\title{
3D STREAMING PROTOCOLS FOR SPATIAL DATA INFRASTRUCTURE: A BRIEF REVIEW
}

\author{
C. B. Siew, A. Abdul Rahman \\ Geospatial Information Infrastructure, Faculty of Geoinformation and Real Estate, Universiti Teknologi Malaysia \\ (UTM), Johor Bahru, Malaysia. \\ bernad@bernadsiew.name, alias@utm.my
}

KEY WORDS: 3D Streaming Protocol; SDI; Area of Interest

\begin{abstract}
:
Web services utilizations in Spatial Data Infrastructure (SDI) have been well established and standardized by Open Geospatial 3D graphics rendering has been a topic of interest among scientific domain from both computer science and geospatial science. Different methods were proposed and discussed in these researches for different domains and applications. Each method provides advantages and trade-offs. Some methods proposed image based rendering for 3D graphics and ultimately. This paper attempts to discuss several techniques from past researches and attempts to propose another method inspired from these techniques, customized for 3D SDI its data workflow use cases.
\end{abstract}

\section{INTRODUCTION}

Streaming protocols are seen essentials for 3D graphics rendering over network environment. This is due to the fact that structured protocols effectively encode data over the network. Different approaches have shown improvements in rendering time, interactive experience, minimizes output distortion and etc, however, on the other hand, suffer quality degradation and packet loss. With current emerging trends in Massive Multiplayer Online Games (MMOGs), as well as the emerging trend in implementations of $3 \mathrm{D}$ in various GIS applications, streaming 3D over the internet is getting common and necessary.

Spatial Data Infrastructure (SDI) for state-wise 3D visualizations and analysis coulBased on previous researches, several streaming protocols are highlighted and discussed in this paper. At the last section of this paper, a brief method is proposed for establishing 3D streaming protocol for spatial data, followed by summary of future directions.

\section{BACKGROUND}

In computer graphics, 3D visualizations of object near to user's viewpoint has been a popular factor of study area. Different culling method were created, resulting different outputs (Moller et. al, 2002) (Zimmerman, 2012). Backface culling eliminates polygons that face away from user view point. View frustum culling eliminates polygons that are located outside of user view frustum.

Occlusion culling determines the removal of groups of polygons in a complex calculation by finding the interactions if they are blocked by other polygons. While these techniques are commonly applicable in local virtual environment, visualization of these 3D objects in SDI falls under Networked Virtual Environment (NVE) category (Aljaafreh M. et, al, 2013).

Utilizing level of details crossing networked environment is practical. Flow Level of Detail (FLoD) proposed by (Hu et. al,
2008) (Shun-Yun et. al, 2010) leverages Peer to Peer (P2P) network delivering level of details of the $3 \mathrm{D}$ objects. Though the concept of Level of Detail (LoD) is different in computer graphic context compared to spatial data standard, e.g. City Geographical Markup Language (CityGML); the idea of view frustum determining level of details could be improvised to allow efficient streaming protocol for spatial data, especially for 3D spatial data such as CityGML.

Object selection protocol (OCTET) proposed by Aljaafreh et al (2013) determines an area of interest, imposing multi-level prioritization based on circle center of the user. This protocol determines that only the objects within the area of interest (AOI) need to be streamed. Headlights in front of user will be subdivided into three zones: a front zone and two border zones. The technique of streaming is using Progressive Mesh (PM) in $3 \mathrm{D}$, a base low resolution layer overlaid by refined resolution layer.

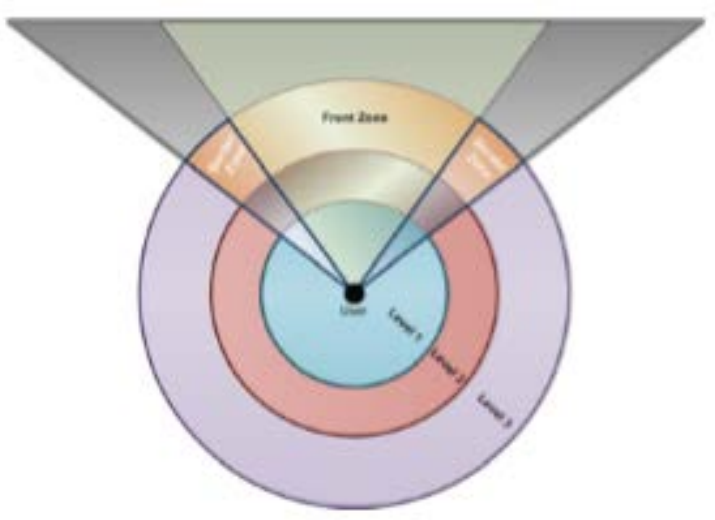

Figure 1. The headlights zoning and area of interest overlapping (Aljaafreh et. al, 2013)

3D model transport protocol (3TP) (Al-Regib and Altunbasak, 2003) uses transport control protocol (TCP) combined with user datagram protocol (UDP). This choice of protocol utilizations depends on three factors: (i) the 3-D models and the sizes of 
connectivity and geometry bitstreams, (ii) the end-to-end channel packet-loss rate, and (iii) the maximum distortion level tolerated by the client. The delay and distortion comparison determine which geometries are selected for TCP and which are for UDP.

\section{AREA OF INTEREST STREAMING PROTOCOL FOR CITYGML}

CityGML classification based on Level of Detail (LoD) could be adopted, given priority for streaming based on area of interest. Headlight zoning dedicated for visualization applications, while user centric circular zoning dedicated for analysis. Progressive transmission on top of priority-based model construction could be defined. For example, which LoD should be delivered based on which zone, link with the progressive tree generated for the objects near the user. This proposed protocol will be based on TCP connection, and it is application layer protocol. User centric object streaming protocol is suitable for application such as navigation, route planning, light weight simulation and analysis, etc.

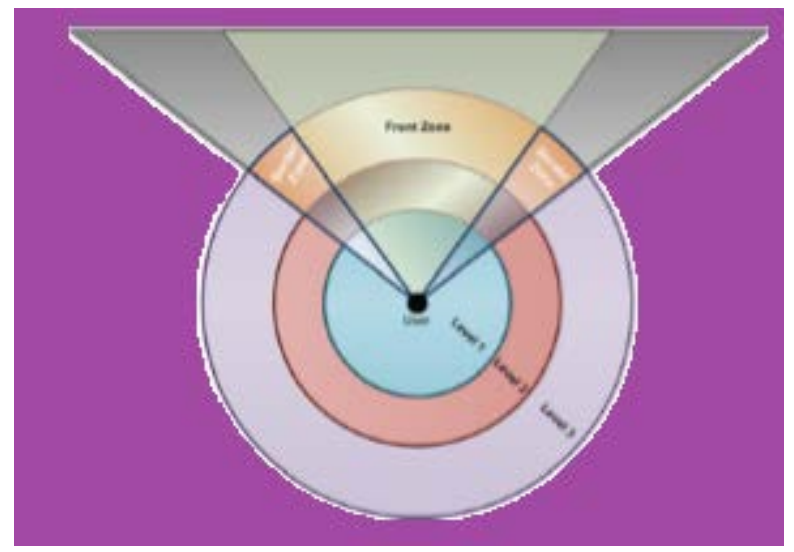

Figure 2: CityGML LoD with area of interest zoning

The front headlight zoning is localized based on inverse pyramid polygon. The circular zoning localization is based on 3D sphere. LoD3.5 to LoD4 streaming should be focused on the front headlight zone. LoD 3 to LoD3.5 on the rear of front head light zone; the purple color zoning is classified for LoD1. For 3D sphere classification, the nearest sphere to user is LoD2.5 to LoD3, second sphere nearest to user is LoD2 to LoD2.5, while third sphere which is the furthest to user is LoD1 to LoD2 and the rest is LoD1.

Based on the area of interest zoning concept, a tree of objects could be constructed based on the priority. Since the retrieval is based on object, semantically the entire object is delivered to client based on the above rule. This protocol is currently prioritized based on area of interest. The delivery of details of the objects depends on the rule in Figure 2.

The advantages of using AOI-based streaming protocol are streaming, progressive, flexible and dynamic in terms of semantic and gemoetric tree information retrieval, and expected to be efficient compared to full CityGML raw retrieval. The virtual scene is reconstructed based on priority, instead of raw retrieval of CityGML flat files or datasets.

With such priority-based AOI streaming method, compression method such as CitySAC (Siew and Abdul Rahman, 2015) could be employed and further improve the efficiency for delivery.

\section{CONCLUDING REMARKS}

In this paper we showcase the draft of 3D streaming protocol for light weight web client. The AOI-based 3D streaming protocol could serve both visualization and light weight analysis. This protocol could be developed at application layer and could be further refined for various SDI applications.

\section{REFERENCES}

Mohammad Aljaafreh, Haifa Raja Maamar, Azzedine Boukerche, 2013, An Efficient Object Discovery and Selection Protocol in 3D Streaming-based Systems over Thin Mobile Devices, IEEE Wireless Communications and Networking Conference (WCNC): Networks, 2013. 978-1-4673-5939-9/13. pp $2393-2398$.

Moller T. Akenine-, T. Moller, E. Haines, Real-Time Rendering, A. K.Peters, Ltd., Natick, MA, USA, 2002.

Zimmermann Seo, R., Quantitative Analysis of Visibility Determinations for Networked Virtual Environments, J. Vis. Commun. Image R. 2012.

S. Hu, T.-H. Huang, S.-C. Chang, W.-L. Sung, J.-R. Jiang, B.Y. Chen, FLOD: A framework for Peer-To-Peer 3d Streaming, Proceedings of IEEE INFOCOM, 2008

Shun-Yun H., J.-R. Jiang, B. Chen, Peer-To-Peer 3D Streaming, IEEE Internet Computing, vol. 14, no. 2, 2010

Ghassan Al-Regib and Yucel Altunbasak, 2003, 3TP An Application-Layer Protocol for Streaming 3-D Graphics. 07803-7965-9/03, 2003, pp 421-424 